\title{
Biotin Ameliorates Muscle Cramps of Hemodialysis Patients: A Prospective Trial
}

\author{
Shiro Oguma, ${ }^{1}$ Itiro Ando, ${ }^{2}$ Takuo Hirose,${ }^{3}$ Kazuhito Totsune, ${ }^{3,4}$ Hiroshi Sekino, ${ }^{5}$ \\ Hiroshi Sato, ${ }^{2}$ Yutaka Imai ${ }^{3}$ and Masako Fujiwara ${ }^{3}$

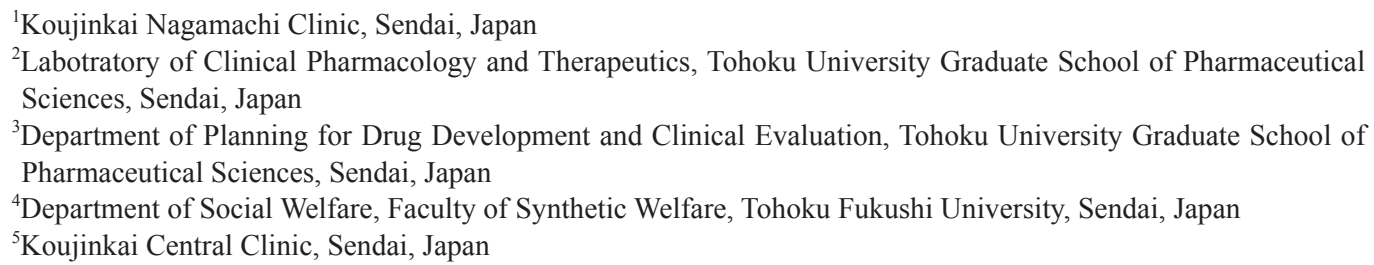

Patients with renal failure undergoing hemodialysis often have muscle cramps during and after the dialysis therapy. Muscle cramps are defined as the sudden onset of a prolonged involuntary muscle contraction accompanied with severe pain, resulting in early termination of a HD session and inadequate dialysis. The etiology of the cramps is unknown and effective anti-cramp medicine is not available. We have hypothesized that water-soluble vitamins are deficient in HD patients. Accordingly, we administrated biotin to 14 patients who had frequent muscle cramps during HD sessions. Oral administration of $1 \mathrm{mg} /$ day biotin promptly reduced the onset and the severity of cramps in 12 patients both during and after HD. Then, the plasma biotin levels were measured by an enzyme-linked immunosorbent assay method (ELISA) in HD patients, including 14 patients with cramps and 13 patients without cramps, and 11 healthy volunteers. Plasma biotin levels were elevated in $27 \mathrm{HD}$ patients at baseline compared with healthy volunteers [451 (377 - 649) vs. 224 (148 - 308) ng/l, median (lower-upper quartiles); $p<0.0001]$. Unexpectedly, among the 14 cramp patients, the biotin levels were significantly higher in biotin-ineffective 7 patients than biotin-effective 7 patients [1,064 (710 - 1,187) vs. $445(359-476) \mathrm{ng} / \mathrm{l} ; p<0.001]$. Thus, the biotins measured by ELISA may consist of not only intact biotin but also its metabolites that do not function as a vitamin. In conclusion, biotin administration is one choice to relieve HD patients from muscle cramps regardless of their elevated plasma biotin levels.

Keywords: biotin; ELISA; hemodialysis; muscle cramp; vitamin

Tohoku J. Exp. Med., 2012, 227 (3), 217-223. (C) 2012 Tohoku University Medical Press

Hemodialysis (HD) patients are susceptible to muscle cramps, which are defined as the sudden onset of a prolonged involuntary muscle contraction accompanied with severe pain, both during dialysis and after returning home (Stewart et al. 1972; McGee 1990; Canzanello and Burkart 1992). Cramps seem to occur more or less about $40 \%$ in HD patients. Since there are now about 300,000 HD patients in Japan, it is estimated that about 120,000 HD patients are suffering from painful cramps in daily HD sessions. All over the world, enormous populations are awaiting for relief from threatening of cramps in HD sessions. A number of palliative measures have been employed but not shown any consistent effects to date (Sakurauchi et al. 1998; Khajehdehi et al. 2001; Hinoshita et al. 2003; Kobrin and Berns 2007; Hung et al. 2009). The origin of this com- plication is likely multifactorial. Subnormal muscle metabolism is thought to be one of the major mechanisms underlying these muscle cramps (Allein et al. 1997). Excessive dialysis ultrafiltration, intradialysis hypotension, or tissue hypoxia may lead to an abnormal utilization of energy by muscles and thus result in muscle cramps (Chillar and Desforges 1972).

In our previous work on proton nuclear magnetic resonance $\left({ }^{1} \mathrm{H}-\mathrm{NMR}\right)$ metabolomics of plasma from $37 \mathrm{HD}$ patients, post-dialysis plasma levels of lactate were increased compared with pre-dialysis plasma levels (Fujiwara et al. 2009). These lactate increments were considered to be a consequence of an energy metabolism disorder in HD patients, and may be caused from any disruptions of the TCA cycle that generates ATP, a major source of

Received April 13, 2012; revision accepted for publication June 15, 2012. doi: 10.1620/tjem.227.217

Correspondence: Masako Fujiwara, Department of Planning for Drug Development and Clinical Evaluation, Graduate School of Pharmaceutical Sciences, Tohoku University, 6-3, Aramaki-aza-aoba, Aobaku, Sendai, Miyagi 980-8578, Japan.

e-mail: fmasako@m.tohoku.ac.jp 
muscle energy. At the entrance of the reactions from pyruvate into the TCA cycle, carboxylase and dehydrogenase accompany the prosthetic groups of biotin and thiamin respectively. We thought that these coenzymes were in shortage in HD patients, because they are small molecules drawn out with water through the dialysis. A part of biotin comes from gastro-bacteria products (Said et al. 1999), we were especially interested in biotin between the two vitamins. The intestinal flora may change in some degree in long-term HD patients who are restricted in dietary intake and affected with drug therapies including antibiotics (Carman et al. 2004). Therefore, we conducted a clinical trial of biotin treatment for 14 HD patients with serious muscle cramps. Plasma biotin levels were measured by an avidin-competitive enzyme-linked immunosorbent assay (ELISA) in HD patients with and without cramps, and compared in relation to the effect of prescribed biotin among cramp patients.

\section{Subjects and Methods}

\section{Patients}

The study protocol was approved by the Ethical Committee of Koujinkai Hospital (Koujinkai Haemodialysis Clinic, Miyagi, Japan). Fourteen HD patients with frequent cramps and 13 HD patients without cramps together with 11 healthy volunteers (non-HD) were recruited, and written informed consent was obtained from them. The trial was conducted according to the principles of the Declaration of Helsinki and in compliance with the regulations of the International Conference on Harmonization/Good Clinical Practice.

The 14 patients with cramps were selected randomly and orally administrated $1 \mathrm{mg}$ /day biotin in the morning without other food restrictions. The mean and standard deviation (S.D.) of the patients was $64.4 \pm 8.5$ (range 55 to 88 ) years old, and 8 were males; they had been dialyzed for the mean of $19.4 \pm 11.4$ (range 4 to 36 ) years. The etiologies of end-stage renal disease were glomerulophritis $(n=7)$, diabetic nephropathy $(n=3)$, IgA nephropathy $(n=1)$ nephrosclerosis $(n=1)$, nephropathy of pregnancy $(n=1)$ and unknown $(n=1)$.

The 13 patients without cramps were selected by possible matching in age, gender, etiology, and HD history with the 14 patients. Their mean age was $65.7 \pm 12.8$ (range 46 to 85 ) years, and 8 were males; they had been dialyzed for $13.8 \pm 9.6$ (range 4 to 36 ) years. The etiologies were glomerulophritis $(n=6)$, diabetic nephropathy $(n=4)$, nephrosclerosis $(n=1)$ and nephrosis $(n=2)$.

The baseline characteristics of the patients are shown in Table 1. The patients with cramps are divided into 2 groups as mentioned bellow. HD treatments were conducted about 4 hours, 3 times a week. The clearance of creatinine is approximately $1 / 3$ in a HD session. Dialysis adequacy expressed as $\mathrm{Kt} / \mathrm{V}$ values was estimated by the single-pool model described by Daugirdas (1993).

The mean ages of 11 healthy controls was $57.5 \pm 10.2$ (range 34 to 69$)$ years old, and 5 were males.

None of the subjects including patients had taken vitamin complex that contains biotin at baseline.

\section{Degree of muscle cramps.}

Muscle cramp was defined as a painful involuntary muscle contraction that lasted more than 1 min during dialysis (McGee, 1990). The intensity of cramps was scored using the following scale: 0 no pain (no early symptoms), 1: mild (under cramps, with early symptoms), 2: discomforting (a cramp, recovery immediate, tolerable), 3: distressing (cramps once or twice, nurse call, recover with some treatment, cramps after HD), 4: horrible and excruciating (several cramps, nurse call, not tolerable, including a cessation of $\mathrm{HD}$, cramps after HD). Each patient was assessed by a written questionnaire of the face-scale (Wong and Baker 1988) with the score above. The score comprises 0 to 4 levels and patients were permitted to specify points midway between scores to describe their status more accurately, thus the results were distributed over 9 levels. An interview to check the effect of the therapy from each patient was carried out 2 weeks after administration of biotin by one of our researchers, not by the physician in charge to avoid flattering bias of patients. It was performed again 6 weeks after administration to confirm the repeatability of the interview.

\section{Interventions: cessation and resumption of biotin treatment}

Among the patients with biotin treatment, 3 patients were persuaded to cease biotin once with the permission of resumption if cramps might relapse again, and two patients had to cease biotin when they changed the other hospital. To sum up, 5 patients were followed in this intervention study.

\section{Measurements of plasma biotin concentration by ELISA}

Venous blood samples from patients and healthy volunteers were collected into lithium heparin tubes. After centrifuging, the plasma was stored at $-25^{\circ} \mathrm{C}$. It was thawed at room temperature immediately before measurements for ELISA (Thuy et al. 1991; Wellenberg and Banks 1993), and any precipitation was removed by centrifugation. Plasma biotin concentration was measured by a competitive enzyme linked assay kit (Immundiagnostik GmbH). The measurements were carried out according to the instructions of the maker.

Plasma samples were collected from 11 healthy adults and 13 patients without cramps at pre-dialysis. Samples from 14 patients with cramps were taken at pre- and post-dialysis, before administration of biotin. Each plasma sample was used at 3 dilutions: 1-fold, 5-fold and 10-fold to be adjusted to optimum concentration for detection. The samples in optimum condition were measured twice and the mean values were used as the concentration of each sample. The intra-assay coefficient of variance was 0.034 .

\section{Statistical analysis}

Statistical analyses were performed with JMP version 5.2. Results are presented as mean \pm S.D. for normally distributed variables and, as median values with lower and upper quartiles for no-normally distributed variable, respectively. Comparisons between groups are given as student- $T$ tests and as Wilcoxon rank sum tests for the corresponding distributions. The chi-square test was performed to compare categorical variables (sex and dialysate buffer) between the 2 groups. Differences of $P<0.05$ are considered to be statistically significant.

\section{Results}

Cramp scores at baseline and the effects of the vitamin administration are summarized in Fig. 1. Before biotin supplementation, 11 patients (1 to 10, and 13) were in a severe distressed state with a higher cramp score, and 3 patients $(11,12$, and 14) were in a distressed state with a 
middle cramp score. The fourteen patients were treated with $1 \mathrm{mg} /$ day of biotin. More than half the patients exhibited remarkable effects within 1 week. After that, stable states were achieved without any side effects. Oral administration of the biotin prevented onset of their cramps during HD and after HD including at night or in the next morning. The effects of biotin were profound $(n=14, P=$ 0.0017).

The results of an intervention study of biotin treatment in 5 patients are shown in Fig. 2; cessation was done at a stationary state 1 month after biotin administration, and resumption (Post-2) occurred several days after cessation because of the relapse of muscle cramp. By the resumption, remission states were achieved again in several days, as well. This intervention represented the reproducibility of biotin effect.

The plasma samples from 27 patients including 14 patients with cramps before administration and 13 patients without cramp together with 11 healthy adults were used for measuring biotin concentration by the ELISA method.

According to the effect of biotin in Fig. 1, we separated the 14 patients into 2 groups as follows: group of 7 patients with relatively significant effect (well-responded group, score of reduction is greater than or equal to 2.0, patients (1 to 7) and group of 7 patients with no or less effect of biotin (poorly-responded group, score of reduction less than 2.0, patients ( 8 to 14 ). Comparison of plasma biotin levels among groups and healthy adults are shown in series of Fig. 3. Fig. 3a shows the biotin levels of 11 healthy adults and 27 patients at pre-HD; the medians and quartiles were $224(148$ - 308) and $451(377$ - 649) ng/l, respectively. Plasma biotin levels of HD patients at baselines were significantly higher than those of healthy adults $(P<0.0001)$, and exhibited a wide distribution with tails upper ward. Fig. 3b shows biotin levels of 13 non-cramp patients and 14 cramp patients; the medians were 430 (366 $649)$ and $601(445-1,029) \mathrm{ng} / \mathrm{l}$, respectively. The cramp patients had also significantly higher plasma levels of biotin than non-cramp patients, $P=0.04$. Non-cramp patients had a tight distribution and the cramp patients had a sparse one. Plasma biotin levels of the well-responded groups and poorly-responded group patients at baseline are shown in Fig. 3c. The medians of plasma biotin levels of the groups of each 7 patients were 445 (359 - 477) ng/l and, 1,064 (710 $1,186) \mathrm{ng} / \mathrm{l}$, respectively. Poorly responded group had significantly higher plasma biotin levels than well-responded group $(P=0.005)$.

In addition, the median of plasma biotin levels in cramp patients at post-dialysis was $354(254$ - 432) ng/l, as shown in Fig. 3d. Plasma biotin levels at pre-dialysis were decreased by HD treatment sufficiently, and the levels after dialysis were even higher than those of healthy people,

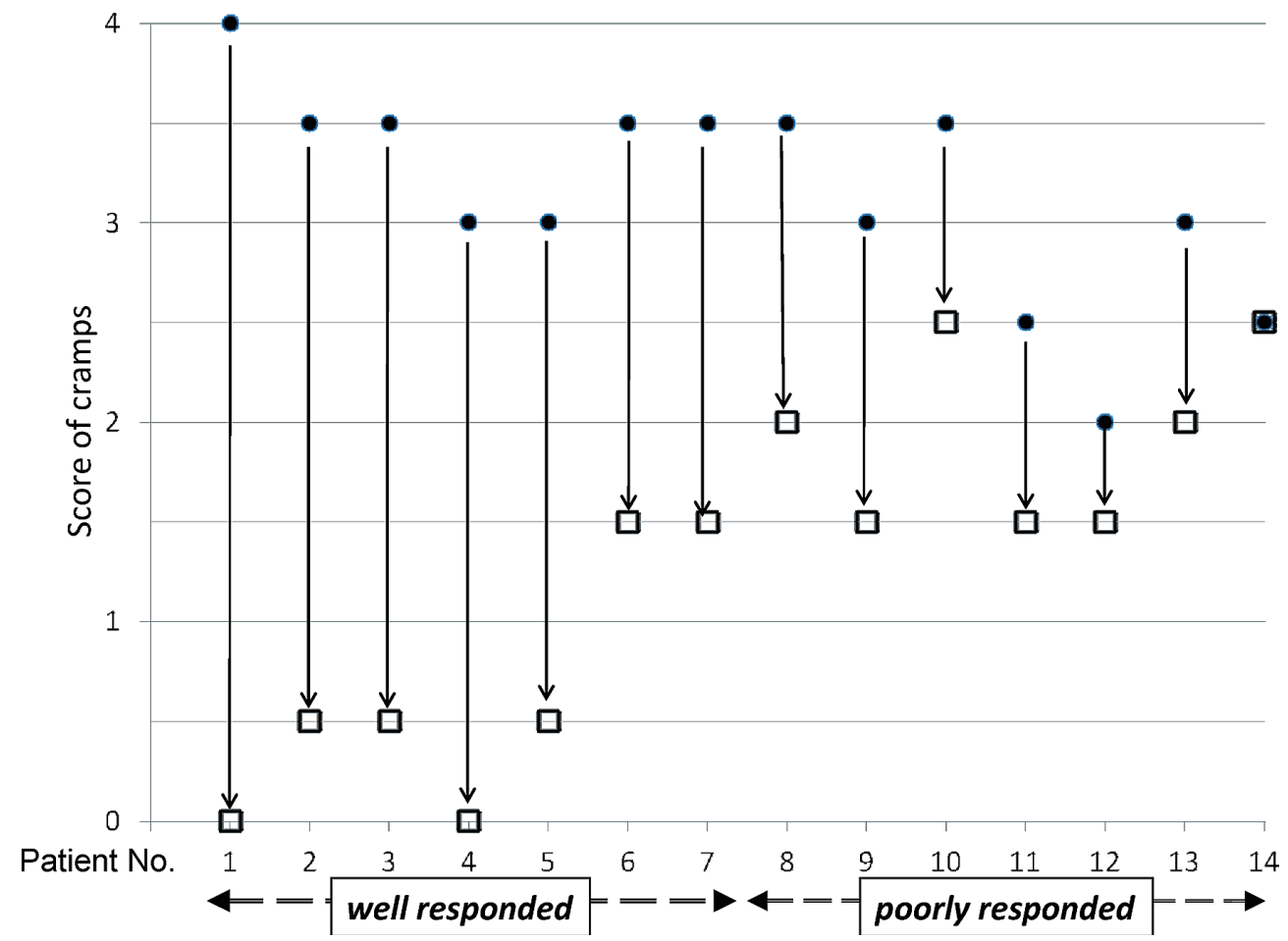

Fig. 1. Intensity score of cramps in 14 patients.

-: at baseline, solid arrows with $\square$ : changes with the end points by biotin. The cramp score uses a scale of no pain, mild, discomforting, distressing, horrible and excruciating, given scores $0-4$, respectively. Twelve patients except No. 12 and 14 exhibited better effects (score reduction more than 1.0) of biotin on cramps. The paired Wilcoxon rank sum tests were $P=0.0017$ for 14 patients between baseline and post-treatment of biotin. The patients are divided into 2 groups, well-responded and poorly-responded, according to the degree of reduction of cramps. 


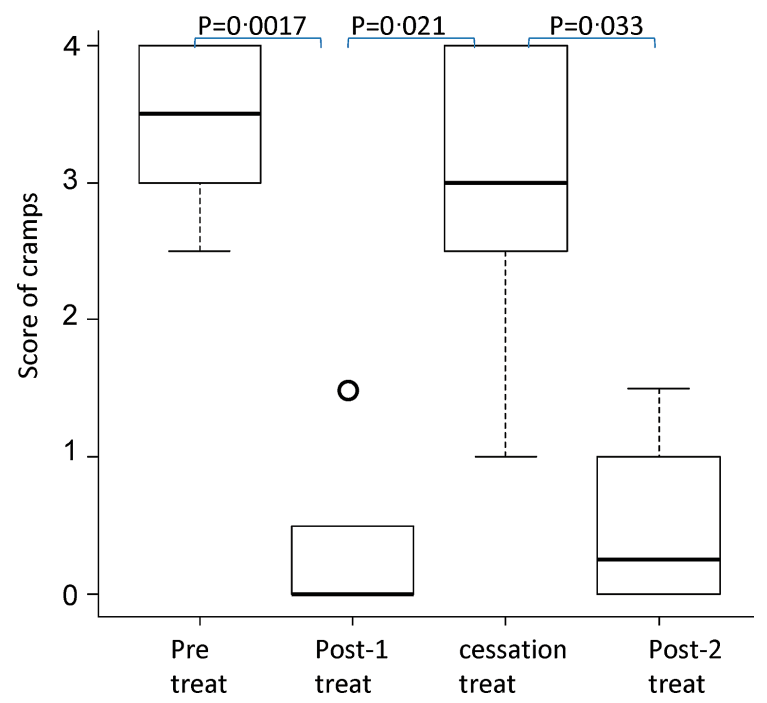

Fig. 2. Box plots showing the intensity scores of cramps in 5 patients.

Biotin treatment (Post-1) ameliorates cramps, and cessation of treatment resulted in relapse of cramps. Resumption treatment (Post-2) reduced cramps again. The circle depicted above the box of Post-1 is an outlier. The paired Wilcoxon rank sum tests are depicted in figure $P=0.0017,0.021$, and 0.033 , respectively.

(a)

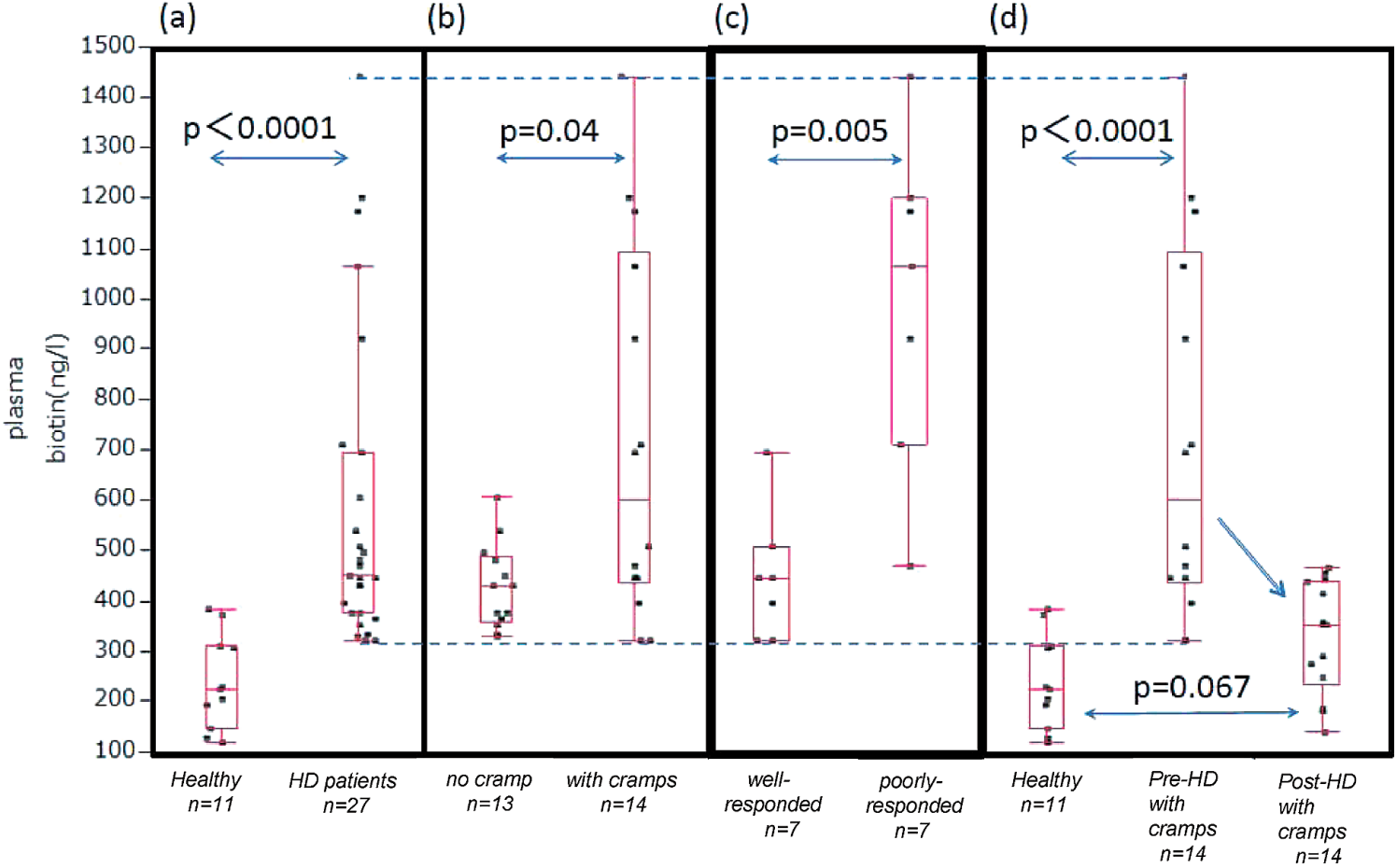

Fig. 3. Box plots of plasma biotin levels measured by an ELISA.

Box plots in each Fig are appended by median, quartiles, minimum and maximum. $P$-values are calculated in Wilcoxon rank sum test. Broken lines through all Figs indicate upper and lower range of patients with cramps.

(a) Comparison between 11 healthy adults and 27 HD patients at pre-dialysis. HD patients (right box) have significantly higher levels than healthy adults $(P<0.0001)$. Their distribution has the tail upper ward with 3 outliers outside the upper whistler.

(b) Comparison between 13 patients without cramp and 14 patients with cramps at pre-dialysis. Patients with cramps have higher biotin levels than those without cramps $(P=0.04)$.

(c) Comparison by efficacy of prescribed biotin on the 14 cramp patients; the group $(n=7)$ with better effect, and the group $(n=7)$ of less effect. The group with poor response has significantly higher levels of biotin than group with well response $(P=0.005)$.

(d) Fourteen cramp patients at pre-dialysis (central box, the same as right box in Fig. 3b), at post-dialysis (right box) and the healthy adults (left box, the same as left box in Fig. 3a). Plasma biotin levels in the patients at post-dialysis were even higher than those of healthy group $(P=0.067)$. 
although not significant $(P=0.067)$.

Biochemical data and dialysis conditions at baseline (pre-prescription of biotin) for 2 groups were summarized in Table 1 together with non-cramp group. Between welland poorly-responded groups, there are no significant differences in biochemical data, age, HD duration and etiology, other than the data of sex, plasma $\mathrm{Mg}$ and creatnine levels. Poorly-responded group included some males with large body mass. There are no significant differences in biochemical data between the cramp patients and noncramp patients

All the data exhibited no significant changes in each group by biotin treatment in 2 months. Plasma lactate levels measured by NMR spectroscopy (method is shown else- where (Fujiwara et al. 2009)) did not change in 14 patients between before and after biotin treatment (data are not shown).

\section{Discussion}

To our knowledge biotin treatment has never been reported as a therapy for the cramps associated with HD until now. In this study, the minimum dose of biotin relieved more than half of $14 \mathrm{HD}$ patients from the pain of muscle cramps.

In our trial we set the biotin dose low for utmost safety. Though no side-effects have been reported concerning even excess administration of biotin to biotin-deficient patients with normal urine excretion (Mock 1996), there

Table 1. Baseline characteristics of study patients $(n=27)$.

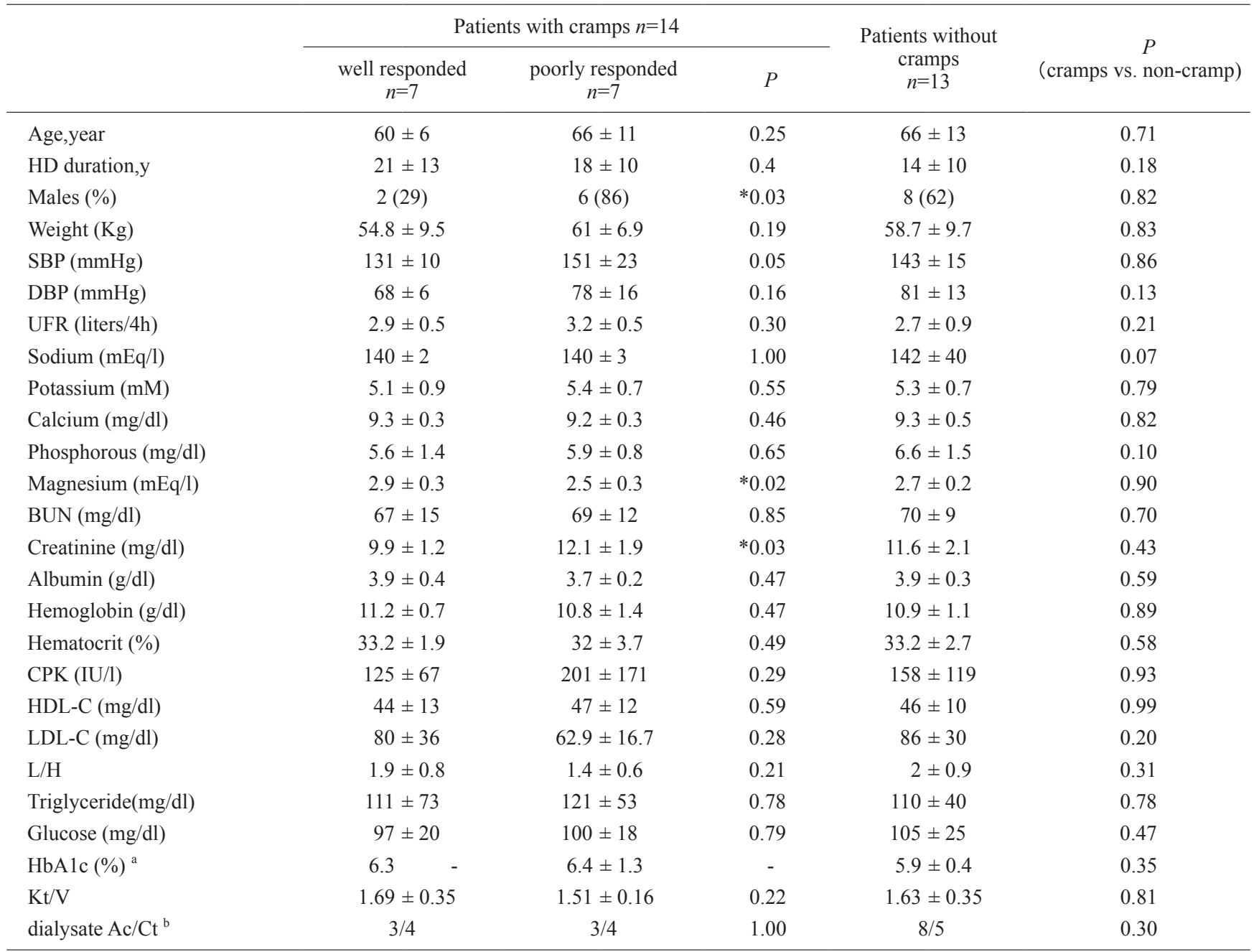

Table shows baseline characteristics of the patients with and without cramps. Patients with cramps are stratified into 2 groups of each $n=7$; group with well-response and group with poor-response. Values are "mean \pm s.D." or "number and percentage". SBP, systolic blood pressures; DBP, diastolic blood pressures; UFR, ultrafiltration rate; BUN, blood urea nitrogen; CPK, creatine phosphokinase; HDL-C, high density lipoprotein cholesterol; LDL-C, low density lipoprotein cholesterol; L/H, the ratio of LDL-C and HDL-C.

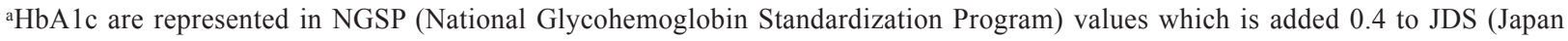
Diabetes Society) values. As the well-responded group includes only one diabetic patient, s.D. of the group and the $P$ value between 2 groups were not calculated. ${ }^{b} \mathrm{HD}$ treatments used bicarbonate dialysis buffers (containing 8-12 $\mathrm{mM}$ acetate and 5-8 $\mathrm{mM}$ glucose) (indicated as $A c$ ), or citrate dialysis buffers (containing $0.7 \mathrm{mM}$ citrate and $8 \mathrm{mM}$ glucose) (indicated as $C t$ ). $*$ indicate the values of $P<0.05$. 
have been no reports about proper biotin dosage for longterm HD patients who are almost anuric. Descombes et al. (1993) reported that anuric HD patients had high levels of plasma biotin, and they concluded there was no need for a biotin supplement. On the contrary, they recommended prescription of other vitamins including thiamine for insufficient range (Kurijima et al. 1980). Our results also exhibited no deficiencies of biotin in the HD patients even at post-dialysis; they had high levels of plasma biotin compared with normal subjects. In addition, HD patients with frequent muscle cramps also had significantly higher plasma biotin levels than patients without cramps at baseline. Nevertheless, it is striking that biotin supplementation had a remarkable effect on cramps, and that the degree of the effects depended on the biotin levels of patients at baseline. The patients with better effect had lower biotin levels, whereas the patients with less efficacy had high biotin accumulation. The significant difference $(P=0.005)$ in plasma biotin levels indicates that biotin levels have an inverse relationship with the effect of prescribed biotin for muscle cramps in HD patients. Considering the distributions of groups (Fig. 3a, b), poorly-responded group was distinctive from both well-responded group and non-cramp group.

Thus HD patients showed higher biotin levels and the paradox that biotin administration had an effect on cramps in the HD patients with abundant biotin levels is not easily explained. One of the possible explanations is the accumulation of biotin metabolites in HD patients. We measured biotin levels in plasma by the ELISA method, a competitive avidin binding assay. Total avidin-binding substances in plasma are not only biotin but also its metabolites such as bisnorbiotin, and biotin sulfoxide, etc (Mock et al. 1993, 1995; Zempleni and Mock 1999), which are not distinguished by the ELISA. Bisnorbiotin and others are catabolized from biotin, and they are reported to have no activity as a co-enzyme (Zempleni et al. 1997). Since these metabolites have structural similarities to biotin, the potential exists that the metabolites compete and interfere with intact active biotin for intestinal or cellular uptake, or delivery to tissues (Said et al. 1987)

Considering this, the patients with cramps may be in deficiency of biotin activity in spite of their apparently abundant plasma biotin levels. We infer that the total active biotin in the well-responded patients remained insufficient and therefore the prescribed biotin worked as an active vitamin. One of the possible mechanisms why the biotin effect was less in poorly-responded patients is that they have too high biotin levels mainly composed of its various metabolites for a low dose of biotin administration to recover its activity. More dose of biotin may overcome the suppression by the metabolites to be activated as a vitamin in poorly-responded group.

These results indicate a possible action of biotin to intervene in the mechanism producing cramps, probably via compensation for subnormal energy metabolism. Muscle energy utilization may be enhanced by the biotin which has a basic importance in the energy generative TCA cycle, on which another candidate cofactor is thiamine. We had several case studies of additional administration of thiamine with better effect (not shown here).

In this study, however, we have no evidence to confirm our hypothesis of biotin-metabolites accumulation in mainly poorly-responded patients. Further investigations are required about the precise composition of biotin in the plasma in HD patients.

In addition, it is unclear why the better effect after biotin administration persisted despite the biotin levels were dropped after HD session. The result suggests that plasma biotin level might be unable to play a pathophysiologic role in HD patients with cramps, although it showed better effect. Therefore, further investigation is required on this issue. Examination of plasma biotin levels and composition after administration in patients stratified with the effect of biotin would be helpful to clarify this issue, although it was not performed in the present study.

It is known that $\mathrm{Ca}^{2+}$ regulates signal transduction pathways on skeletal muscle (Zaidi et al. 1989; Rorseman et al. 2000) and Ca solution has been used to treat muscle cramp patients, however we have no data to discuss the effect of biotin on the $\mathrm{Ca}^{2+}$ regulation in this small-sized study.

In conclusion, the present study demonstrated that the biotin treatment is one of the choices to treat HD patients with muscle cramps. We expect further long-term, large scale studies to reconfirm the effects and safety of biotin therapy for the cramps of HD patients.

\section{Acknowledgments}

This research was in part supported by Grants-in-Aids from the Ministry of Education, Culture, Sports, Science and Technology of Japan (22590035 and 23790242), and from Miyagi Kidney Foundation on 2011. We thank staffs of Koujinkai Nagamachi Clinic, who collected samples from patients and gave us information of patients.

\section{Conflict of Interest}

We declare no interest.

\section{References}

Allein, S., Majumdar, S., De Bisschop, E., Newitt, D.C., Luypaert, R. \& Eisendrath, H. (1997) In vivo comparison of MR phase distribution and $1 / \mathrm{T} 2 *$ with morphologic parameters in the distal radius. J. Magn. Reson. Imaging, 7, 389-393.

Canzanello, V.J. \& Burkart, J.M. (1992) Hemodialysis-associated muscle cramps. Semin. Dial., 5, 200-304.

Carman, R.J., Simon, M.A., Fernández, H., Miller, M.A. \& Bartholomew, M.J. (2004) Ciprofloxacin at low levels disrupts colonization resistance of human fecal microflora growing in chemostats. Regul. Toxicol. Pharm., 40, 319-326.

Chillar, R.K. \& Desforges, J.F. (1972) Muscular cramps during maintenance haemodialysis. Lancet, 2, 285.

Daugirdas, J.T. (1993) Second generation logalithmic estimations of single-pool variable volume $\mathrm{Kt} / \mathrm{V}$ : an analysis of error. $J$. Am. Soc. Nephrol., 4, 1205-1213.

Descombes, E., Hanck, A.B. \& Fellay, G. (1993) Water soluble 
vitamins in chronic hemodialysis patients and need for supplementation. Kidney Int., 43, 1319-1328.

Fujiwara, M., Kobayashi, T., Jomori, T., Maruyama, Y., Oka, Y., Sekino, H., Imai, Y. \& Takeuchi, K. (2009) Pattern recognition analysis for ${ }^{1} \mathrm{H}$ NMR spectra of plasma from hemodialysis patients. Anal. Bioanal. Chem., 394, 1655-1660.

Hinoshita, F., Ogura, Y., Suzuki, Y., Hara, S., Yamada, A., Tanaka, N. \& Yamashita, A. (2003) Effect of orally administered shaoyao-gan-cao-tang (Shakuyaku-kanzo-to) on muscle cramps in maintenance hemodialysis patients: a preliminary study. Am. J. Chin. Med., 31, 445-453.

Hung, C.Y., Chen, Y.L., Chen, C.S., Yang, C.S. \& Peng, S.J. (2009) Association of leptin with hemodialysis-related muscle cramps: a cross-sectional study. Blood Purif., 27, 159-164.

Khajehdehi, P., Mohammad, M., Behzadi, S. \& Rais-Jalali, G. (2001) A randomized, double-blind, placebo-controlled trial of supplementary vitamins E, C and their combination for treatment of haemodialysis cramps. Nephrol. Dial. Transplant., 16, 1448-1451.

Kobrin, S.M. \& Berns, J.S. (2007) Quinine-a tonic too bitter for hemodialysis-associated muscle cramps? Semin. Dial., 20, 396-401.

Kurijima, M., Mizuta, A., Yokomine, R., Igata, A. \& Otuji, Y. (1980) Erythrocyte transketolase activity in uremia. Clin. Chim. Acta, 108, 169-177.

McGee, S.R. (1990) Muscle cramps. Arch. Intern. Med., 150, 511-518.

Mock, D.M., Lanford, G.L. \& Cazin, J. (1993) Biotin and biotin analogs in human urine: biotin accounts only half of the total. J. Nutr., 123, 1844-1851.

Mock, D.M., Lanford, G.L. \& Mock, N.I. (1995) Biotin accounts for only half of the total avidin-binding substances in human serum. J. Nutr., 125, 941-946.

Mock, D.M. (1996) Biotin. In: Ziegler, E.E., Filer, L.J. Jr., eds. Present knowledge in Nutrition, $7^{\text {th }}$ ed. Washington, DC: ILSI
Nutrition Foundation, pp. 220-235.

Rorseman, P., Eliasson, L., Renstom, E., Gramada, J., Barg, S. \& Göpel, S. (2000) The cell physiology of biphasic insulin secretion. News Physiol. Sci., 15, 72-77.

Said, H.M., Redha, R. \& Nylander, W. (1987) A carrier-mediated, $\mathrm{Na}^{+}$gradient-dependent transport for biotin in human intestinal brush-border membrane vesicles. Am. J. Physiol., 253, G631- G636.

Said, H.M. (1999) Cellular uptake of biotin: mechanisms and regulation. J. Nutr., 129, 490S-493S.

Sakurauchi, Y., Matsumoto, Y., Shinzato, T., Takai, I., Nakamura, Y., Sato, M., Nakai, S., Miwa, M., Morita, H., Miwa, T., Amano, I. \& Maeda, K. (1998) Effects of L-carnitine supplementation on muscular symptoms in hemodialyzed patients. Am. J. Kidney Dis., 32, 258-264.

Stewart, W.K., Fleming, L.W. \& Manuel, M.A. (1972) Muscle cramps during maintenance haemodialysis. Lancet, 1, 10491051.

Thuy, L.P., Sweetman, L. \& Nyhan, W.L. (1991) A new immunochemical assay for biotin. Clin. Chim. Acta, 202, 191-197.

Wellenberg, G.J. \& Banks, J.N. (1993) Enzyme-linked sorbent assay to quantify $d$-biotin in blood. J. Sci. Food Agric., 63, $1-5$.

Wong, D.L. \& Baker, C.M. (1988) Pain in children: comparison of assessment scales. Pediatr. Nurs., 14, 9-17.

Zaidi, N.F., Lagenaur, C.F., Hilkert, R.J., Xiong, H., Abramson, J.J. \& Salama, G. (1989) Disulfide linkage of biotin identifies a $106-\mathrm{kDa} \mathrm{Ca}^{2+}$ release channel in sarcoplasmic reticulum. $J$. Biol. Chem., 264, 21737-21747.

Zempleni, J., McCormick, D.B. \& Mock, D.M. (1997) Identification of bisnorbiotin methyl ketone, and tetranorbiotin- $l$-sulfoxide in human urine. Am. J. Clin. Nutri., 65, 508-511.

Zempleni, J. \& Mock, D.M. (1999) Biotin biochemistry and human requirements. J. Nutri. Biochem., 10, 128-138. 